\title{
Xenon Upregulates Hypoxia Inducible Factor 1 Alpha in Neonatal Rat Brain under Normoxic Conditions
}

\author{
Simona Valleggi, ${ }^{1}$ Chirag B. Patel, ${ }^{2}$ Andrea O. Cavazzana, ${ }^{3}$ Daqing Ma, ${ }^{4}$ \\ Francesco Giunta, ${ }^{5}$ and Davide Cattano ${ }^{2}$ \\ ${ }^{1}$ Department of Physiology and Biochemistry, School of Medicine, University of Pisa, Pisa, Italy \\ ${ }^{2}$ Department of Anesthesiology, University of Texas Medical School at Houston, 6431 Fannin Street, Houston, TX 77030, USA \\ ${ }^{3}$ Division of Surgical Molecular and Ultrastructural Pathology, School of Medicine, University of Pisa, Pisa, Italy \\ ${ }^{4}$ The Anaesthetics, Pain Medicine, and Intensive Care Section, Department of Surgery and Cancer, Imperial College London, \\ Chelsea \& Westminster Hospital, London, UK \\ ${ }^{5}$ Surgical Pathology Unit, Department of Oncology, ASL no. 1, Massa-Carrara, Italy
}

Correspondence should be addressed to Davide Cattano, davide.cattano@uth.tmc.edu

Received 20 October 2011; Accepted 9 November 2011

Academic Editors: J.-H. Baumert and C.-T. Wu

Copyright (C) 2011 Simona Valleggi et al. This is an open access article distributed under the Creative Commons Attribution License, which permits unrestricted use, distribution, and reproduction in any medium, provided the original work is properly cited.

Xenon can induce cell and organ protection through different molecular mechanisms related to oxygen level. We explored the effect of xenon on oxygen-related signalling in the central nervous system via hypoxia inducible factor 1 alpha (HIF- $1 \alpha)$ and mammalian target of rapamycin (mTOR). Methods. Postnatal day 7 (P7) Sprague Dawley rats were exposed to 25\% oxygen/75\% nitrogen (air group) or 25\% oxygen/75\% xenon (treatment group) for 120 min. Brains were collected immediately (transcript analysis-relative real-time polymerase chain reaction) or 24 hours (protein analysis-immunohistochemistry) after the 120-minute exposure period; peak anesthetic preconditioning has been previously identified at 24 hours post-exposure. Results. HIF- $1 \alpha$ transcript and protein levels were found to be increased in xenon-exposed compared to air-exposed brains. Sustained nuclear translocation of the protein, accounting for an increased activity of HIF- $1 \alpha$, was also noted. mTOR transcript analysis revealed no significant difference between xenon-exposed and air-exposed brains immediately after the 120-minute exposure. Conclusion. Our data suggest that xenon induces the upregulation of HIF- $1 \alpha$ transcription and translation, which may contribute to xenon's neuroprotective preconditioning effect. However, given that xenon exposure did not affect mTOR transcription, further investigation into other signalling cascades mediating xenon's effects on HIF- $1 \alpha$ in developing brain is warranted.

\section{Introduction}

Xenon has been approved in Europe as a medical gas, and its utility has been demonstrated through clinical and basic research studies. Although major interest focuses on xenon's hemodynamic and analgesic properties, increasing interest has recently focused on its potential long-lasting neuroprotective properties, which have been demonstrated both in in vivo and in vitro models of neuronal injury [1-5]. Similar properties have been observed in the setting of anesthetic preconditioning [6-8], suggesting that xenon may play a useful clinical role in therapeutically unmet disease states such as stroke or neonatal asphyxia. Preconditioning is a biological process observed in several organs, in which a transient stimulus induces a protective state against a more prolonged, potentially lethal insult $[9,10]$. Experimental studies into forms of anesthetic-induced preconditioning (APC), including hypoxia and sublethal ischemia, have indicated that preconditioning occurs in two waves (early and delayed) and with two potencies (short/immediate and long/deferred) [11]. The early phase, mostly comprising phosphorylation steps, starts immediately after the application of the preconditioning stimulus and lasts for 2-3 hours [12], while the delayed phase is evident after 12-24 hours and lasts for up to 3 days. This long-lasting effect is the result of a transient alteration in gene transcription and depends on protein synthesis 
$[13,14]$. Because it seems to be agent-, organ-, species-, and age-dependent $[13,15-17]$, further investigation into the mechanisms of preconditioning is warranted.

Studies of asphyxia and hypoxia-ischemia, and also isoflurane toxicity and apoptosis, suggest that xenon may be a valuable preconditioning and neuroprotective anesthetic agent that does not exert neurotoxic effects in the fragile developing brain $[1,7,8,16-18]$. Xenon induces early transcriptional changes in the brain of neonatal rodents exposed for 2 hours to the gas $[19,20]$, and although an increase in mRNA does not always result in new protein synthesis, we previously demonstrated an increase in both signal and message of activity-dependent neuroprotective protein (ADNP), a key brain protein involved in several cell survival mechanisms, 24 hours after xenon exposure (the peak of delayed preconditioning) [19].

Hypoxia inducible factor 1 alpha (HIF- $1 \alpha$ ) is regulated by cellular oxygen tension [21] and is important in neuronal protection during hypoxia/ischemia and delayed preconditioning events. One of the signals upstream of HIF- $1 \alpha$ is the mammalian target of rapamycin (mTOR), which regulates many activities including cell proliferation, cell survival, and protein synthesis; mTOR is inhibited by rapamycin [22]. Although the effects of HIF- $1 \alpha$ in xenon-mediated preconditioning have been confirmed extensively in an adult mouse model of kidney ischemia [23], the role of xenon-induced preconditioning in the developing brain remains unknown.

Given the importance of HIF- $1 \alpha$ in mediating preconditioning events and the incomplete understanding of xenon's mechanism of action, our aim was to study the possible role of the mTOR-HIF- $1 \alpha$ signalling axis in xenon-induced preconditioning. Using suppression subtractive hybridization (SSH), relative real-time polymerase chain reaction (PCR), and immunohistochemistry, the effects of xenon exposure on mTOR and HIF- $1 \alpha$ transcript and protein levels were assessed during the peak of delayed preconditioning ( 24 hours postexposure).

\section{Materials and Methods}

2.1. Animal Handling. This study was approved by the Animal Welfare Committee at the University of Pisa, and experiments were carried out in accordance with the European Communities Council Directive of 24 November 1986 (86/ $609 / \mathrm{EEC}$ ). All efforts were made to minimize animal suffering and the number of animals used. Twenty P7 SpragueDawley rats from two different litters were used. The two cohorts, xenon-exposed rats (experimental) and air-exposed rats (control), were randomly assigned ( $n=10 /$ cohort). The control group was exposed to $25 \%$ oxygen in $75 \%$ nitrogen (air group), and the treated group was exposed to 25\% oxygen in $75 \%$ xenon. Xenon concentration was measured continuously by an in-line gas analyzer (Air Products model no. 439Xe, Allentown, PA), while other gases were monitored through infrared gas analyzer (Datex Inc., Helsinki, Finland). The temperature of the exposure chambers was maintained at $37^{\circ} \mathrm{C}$ via a water bath. After 2 hours of exposure, $n=6$ rats from each cohort were immediately decapitated, and their forebrains were isolated in ribonuclease-free conditions, snap frozen in liquid nitrogen, and stored at $-80^{\circ} \mathrm{C}$.

2.2. RNA Extraction. Total ribonucleic acid (RNA) was isolated from the forebrains as previously described [20]. Tissue samples were homogenized in an Ultra-Turrax homogenizer (IKA Works, Inc. Wilmington, NC) in the presence of a denaturing guanidium thiocyanate buffer. Homogenates were laid over a cesium chloride cushion and ultracentrifuged at 40,000 rpm for 24 hours (Beckman Ultracentrifuge L8-70 M; rotor Kontron TY65). The RNA pellet was dissolved in DEPC-treated water, precipitated overnight with 0.1 volume $(\mathrm{v} / \mathrm{v})$ of sodium acetate $(3 \mathrm{M}, \mathrm{pH}$ of 6.4$)$ and 2.2 volumes $(\mathrm{v} / \mathrm{v})$ of absolute ethanol, and solubilized in DEPC-treated water. The concentration and purity of isolated RNAs was determined spectrophotometrically (optical density 260/280 ratio between 1.8 and 2.0). For quality control purposes, total RNA was denatured with formaldehyde and size-fractionated through a $1 \%$ denaturing agarose gel, and visualized by ethidium bromide staining of the gel $(28 \mathrm{~S} / 18 \mathrm{~S}$ ratio between 1.5 and 2.5). The samples were then stored at $-80^{\circ} \mathrm{C}$. Poly(A) + RNAs were isolated from the pools of total RNAs using the PolyATtract mRNA Isolation Kit (Promega, Madison, WI) according to manufacturer's protocol. Poly(A) + mRNA quality and integrity were assessed by RT-PCR (ImProm-II Reverse Transcription System, Promega, Madison, WI) using 3 specific primer sets for each recombinant deoxyribonucleic acid (DNA) gene (18S, $5.8 \mathrm{~S}$, and 28S, resp.) and one glyceraldehyde-3-phosphate dehydrogenase-(G3PDH-) specific primer set.

\subsection{Suppression Subtractive Hybridization. Suppression sub-} tractive hybridization (SSH) was performed using the $\mathrm{BD}$ PCR-Select complementary DNA (cDNA) Subtraction Kit (BD Biosciences-Clontech, Palo Alto, CA) according to the manufacturer's protocol. The procedure required $0.5-1 \mu \mathrm{g}$ of poly(A) + RNA from mRNA populations of both groups that was first converted to cDNAs. The cDNAs were used as tester (samples from xenon-exposed group) and driver (samples from control group) for the forward subtraction and vice versa for the reverse subtraction. Two cDNA libraries were thus obtained: the forward library containing genes positively modulated or switched on by xenon exposure and the reverse library containing genes negatively modulated or switched-off by xenon exposure. Tester and driver cDNAs were hybridized, and the hybrid sequences were removed. Consequently, the unhybridized cDNAs represented fragments corresponding to modulated or on/off switched sequences. The efficiency of subtraction was estimated by hybridization and PCR analyses. Hybridization analyses were carried out with Southern blot of cDNAs derived from subtracted and unsubtracted libraries. cDNAs derived from forward-and reverse-subtracted libraries were used as probes after being labelled with digoxigenin (DIG) DNA Labelling Kit (Roche, Mannheim, Germany). PCR analysis consisted of amplification of the G3PDH reference gene cDNA in subtracted and unsubtracted cDNAs, in order to evaluate its differential expression in subtracted and unsubtracted samples. Aliquots of the samples were drawn after 18, 23, 28, 
and 33 cycles of PCR amplification, and the products were then analyzed by agarose gel electrophoresis. Amplified cDNA sequences from the forward library were then directly inserted into a T/A-cloning vector using the TOPO TA Cloning Kit (Invitrogen, Milan, Italy). Because the final SSH products were enriched but not strictly composed of differentially expressed cDNAs, a differential screening was performed in order to sort out false positive clones. Positive clones were PCR amplified by colony PCR and then checked by $2 \%$ agarose gel electrophoresis. $1 \mu \mathrm{L}$ of each heat-denatured PCR product was spotted onto positively charged nylon membrane (Roche, Mannheim, Germany) and UV crosslinked by Hoefer UVC 500 Ultraviolet Cross-linker (Amersham Biosciences, Piscataway, NJ). Two identical blots were created and hybridized with DIG-labelled forward and reverse library probes. In order to quantify the difference in signal intensity, spots were scanned using an image scanner, and signals were quantified with the Image Master 2D platinum software version 5.0 (Amersham Biosciences, Piscataway, NJ). Clones were subjected to differential screening, and those showing an on/off hybridization signal were sequenced by the dideoxy chain terminator method with an automated DNA sequencer (MGM, Institute of Molecular Genetics and Medicine, Pisa, Italy). Database searches were performed using the National Center of Biotechnology Information server (http://www.ncbi.nlm.nih.gov/) with BLAST algorithms at the European Bioinformatics Institute server (http://www.ebi.ac.uk/) and at the Rat Genome Database (http://rgd.mcw.edu/).

2.4. Data Validation and Expression Analysis. SSH raw data validation was performed using relative RT-PCR on total RNA used for construction of libraries. PCR products were separated on $2 \%$ agarose gel electrophoresis, and band intensities were analyzed by Quantity One software version 4.4.1. (Bio-Rad, Hercules, CA). Validation of selected sequences and expression analysis were performed on the other (validation) animal cohort with relative real-time PCR (MGM, Institute of Molecular Genetics and Medicine, Pisa, Italy) using 7900HT FAST Real-Time PCR Sybr Green I Dyemethod (Applied Biosystems, Foster City, CA) according to the manufacturer's instructions. Expression analysis of mTOR transcript was performed using semiquantitative RTPCR on RNA from the validation set (Cyclophylin A used as reference gene). Cyclophylin A fragment amplification length was $351 \mathrm{bp}$, and mTOR fragment amplification length was 409 bp. Specific PCR primers were designed using Primer3 software (http://frodo.wi.mit.edu/primer3/) and subjected to a BLAST search to ensure that the sequences did not share high homology with identified mRNAs. $100 \mathrm{ng}$ of total RNA was used for reverse transcription using ImProm-II Reverse Transcription System (Promega, Madison, WI) and Oligo(dT) 15 primers according to the manufacturer's protocol. $1 \mu \mathrm{L}$ of $1: 10$ diluted reverse transcript was used as the PCR template. PCR products were separated with $2 \%$ agarose gel electrophoresis, and band intensities were analyzed by Quantity One software version 4.4.1. (Bio-Rad, Hercules, CA).
2.5. Immunohistochemistry. Immunohistochemistry was performed on $n=4$ rats/cohort to assess for relationships between gene transcription, protein translation, and localization at the cellular level. Twenty-two hours after the 2-hour xenon or air exposure, rats were euthanized with thiopental intraperitoneal injection $(100 \mathrm{mg} / \mathrm{kg}$ body weight $)$ and perfused with $4 \%$ paraformaldehyde. Brains were removed, postfixed overnight in perfusate, and processed for paraffin embedding. Serial coronal sections ( $6 \mu \mathrm{m}$ thickness) were cut with a microtome, collected on super-frost slides, and dried overnight at $37^{\circ} \mathrm{C}$. Paraffin sections were stained with antibodies against HIF- $1 \alpha$ (ab1, 1:100 dilution, Abcam, Cambridge, UK). Visualization of primary antibody was achieved with VECTASTAIN Elite ABC Kit (Vector Laboratories, Burlingame, CA) and diaminobenzidine tetrahydrochloride as substrate (K3466, DakoCytomation, Denmark), a technique based on the indirect streptavidin-biotin method. Slides were counterstained with hematoxylin. Staining intensity and morphologic qualitative assessment were carried out by two blinded independent observers using 20x and 40x magnification in two separate evaluation areas for each animal. Images were captured with the same exposure time for both groups at each magnification level.

2.6. Statistical Analysis. Statistical analysis of real-time PCR data was performed using REST 2005 version 1.9.12 software (Corbett Research, Sydney, Australia). Analysis of relative mTOR/Cyclophilin A transcript from quantified real-time PCR gel images was performed using two-tailed Student's $t$-test (GraphPad Prism 4.00 software, GraphPad Software, La Jolla, CA). Statistical significance was defined as $P<$ 0.05 . Data are reported as mean \pm standard deviation unless otherwise specified.

\section{Results and Discussion}

3.1. Results. Immunohistochemistry analysis of striatum sections from brains obtained 24 hours after the 2-hour exposure period revealed increased HIF- $1 \alpha$ protein synthesis after xenon exposure (Figure 1). In sections from the control group, HIF-1 $\alpha$ immunoreactivity was light in the neuronal cytoplasm and apparently absent in the neuronal nuclei. In sections from the xenon-exposed group, the presence of a strong and diffuse neuronal nuclear HIF- $1 \alpha$ immunoreactivity was detected. Based on the morphological assessment of two independent experts (a neuropathologist and an anatomical histologist), this signal was clearly predominant in neurons and absent from glial cells.

During SSH, after the forward library was inserted into a T/A-cloning vector, 2208 white colonies were obtained. The differential screening of data obtained from SSH technique resulted in the selection of 43 positive clones. Their inserts were sequenced, and in silico analysis was performed using an online database as previously described [20]. Based on its putative role in the cellular response to hypoxia and its suggested neuroprotective properties, the HIF- $1 \alpha$ sequence was subjected to a more detailed analysis. There was a significant 2.3 -fold upregulation in HIF- $1 \alpha$ in the developing brains 


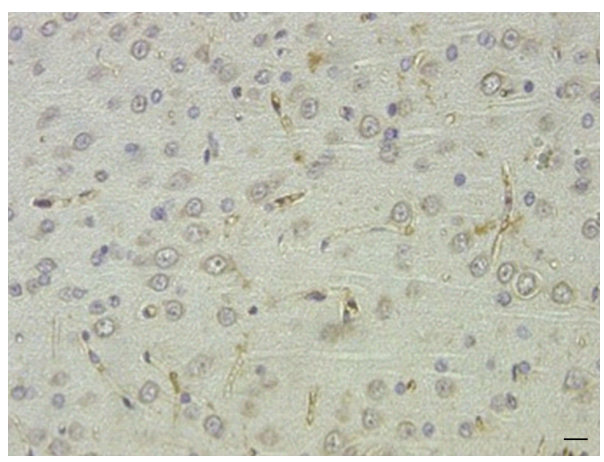

(a)

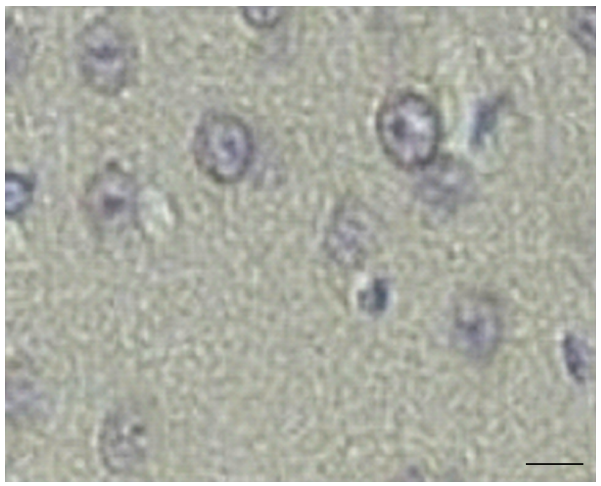

(c)

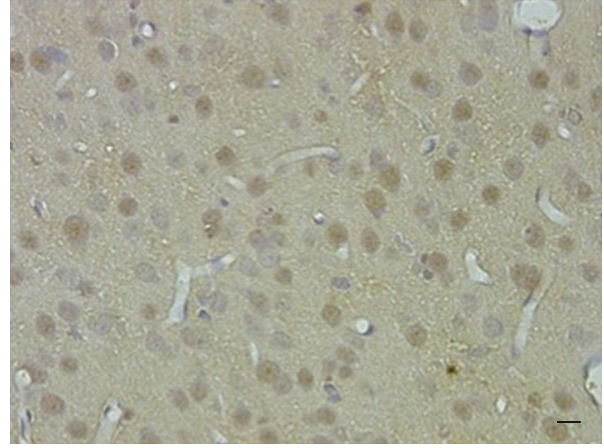

(b)

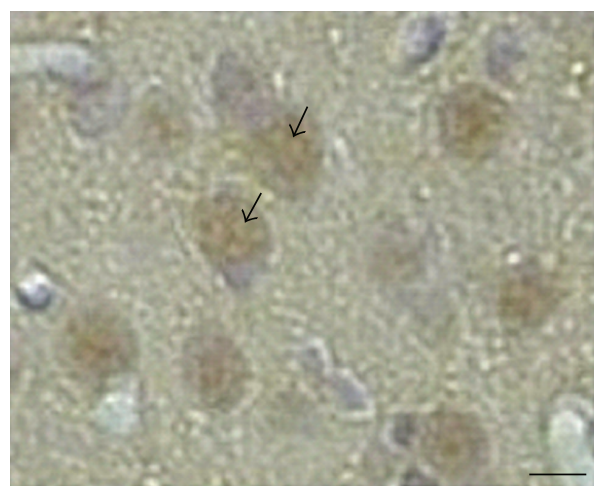

(d)

FIGURE 1: Hypoxia inducible factor 1 alpha (HIF-1 $\alpha$ ) immunohistochemistry in striatum 24 hours following 2-hour exposure period. Immunohistochemistry on paraffin-embedded serial coronal sections ( $6 \mu \mathrm{m}$ thickness), $n=2$ sections/animal, $n=4$ animals/group. For each magnification level, images were captured with the same exposure time for both groups; representative images are shown. (a) Control group brain $(20 \mathrm{x})$ reveals light immunoreactivity in neuronal cytoplasm and absent immunoreactivity in neuronal nuclei. (b) Xenon group brain $(20 \mathrm{x})$ reveals more immunoreactivity in neuronal cytoplasm compared to (a), and immunoreactivity in neuronal nuclei is present. (c) Control group brain (40x). (d) Xenon group brain (40x) reveals strong and diffuse nuclear immunoreactivity (arrowheads) with cytoplasmatic immunoreactivity. Scale bar represents $20 \mu \mathrm{m}$.

of xenon-exposed rats compared to air-breathing animals immediately after the 2-hour exposure period, $P<0.005$ (Figure 2).

In addition, the mTOR sequence was analyzed in detail due to its upstream relation to HIF- $1 \alpha$ and its inhibition with rapamycin leading to loss of xenon-induced preconditioning through HIF- $1 \alpha$. A representative RT-PCR gel showing the amount of mRNA product of mTOR and Cyclophilin A in response to gas exposure is shown in Figure 3. Quantitative gene expression of RT-PCR analysis revealed a mTOR/ Cyclophylin A ratio of $0.67 \pm 0.32$ in the control group and $0.57 \pm 0.36$ in the xenon-exposed group $(P<0.44)$, indicating a lack of xenon effect on mTOR transcription after 2 hours of xenon exposure.

3.2. Discussion. Hypoxic preconditioning refers to a brief period of hypoxia that induces tolerance to brain ischemia and that protects against lethal insults that occur minutes to days following hypoxia. This phenomenon has been related to the synthesis of new RNA and proteins, as well as the posttranslational modification of proteins, and several experimental studies suggest that ischemic tolerance induced by hypoxic preconditioning is due in part to hypoxic induction of HIF- $1 \alpha$ and its target genes [24]. The anesthetic gas xenon has been shown to have preconditioning effects (e.g., protecting cells from late ischemic and hypoxic events) in different preclinical and clinical settings $[15,25]$. We focused our investigation on HIF- $1 \alpha$ not only because it is the main hypoxia sensor in mammalian cells and activates oxygen level-dependent transcription, but also because it is the most important mediator of hypoxia preconditioning [24]. Its transcriptional activity makes HIF- $1 \alpha$ a major contributor to preconditioning in neonatal rat brain [26] and rat retinal neurons [27]. We performed SSH to evaluate the effects of xenon-induced preconditioning in vivo in developing rat brain, under conditions that have previously resulted in preconditioning $[16,20]$. A significant increase in HIF- $1 \alpha$ mRNA transcript in response to xenon exposure was found in our previous study [20], and in the present study we confirm previous findings that xenon also upregulates HIF- $1 \alpha$ protein synthesis [28], which is likely due to at least two signalling axes. The first axis involves the phosphoinositide 3-kinase (PI3K)-Akt-mTOR signalling pathway $[28,29]$ while the second axis involves the mitogenactivated protein kinase (MAPK)-mitogen-activated protein 


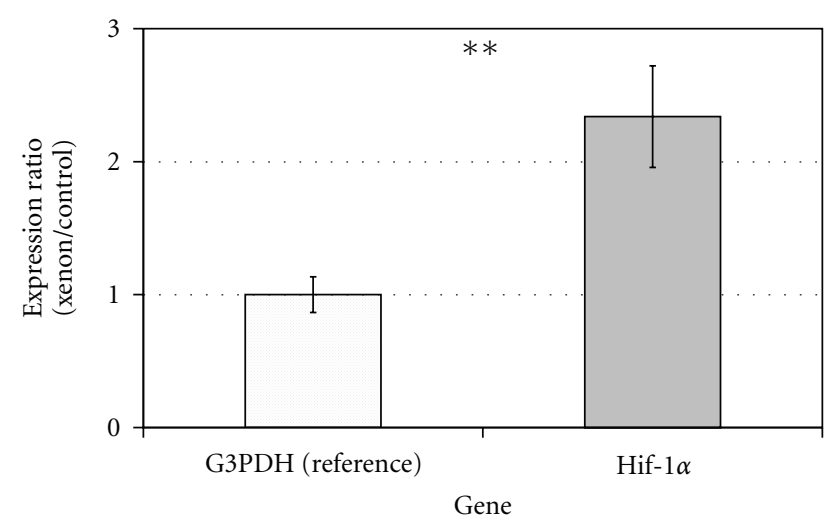

Figure 2: Expression ratio (xenon to control) of hypoxia inducible factor 1 alpha (HIF-1 $\alpha$ ). Compared to air-breathing animals, animals exposed to $75 \%$ xenon for 120 min demonstrated a significant 2.3 -fold increase in HIF- $1 \alpha$ transcript $\left({ }^{* *} P<0.005\right)$ with no increase in reference gene (glyceraldehyde-3-phosphate dehydrogenase $(\mathrm{G} 3 \mathrm{PDH})$ ) expression (reverse transcription polymerase chain reaction analysis). Error bars represent standard error of the mean.

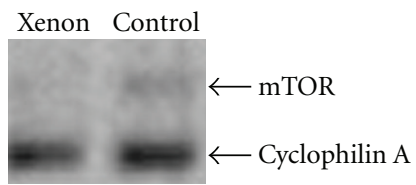

FIGURE 3: Reverse transcription polymerase chain reaction (RTPCR) expression analysis. 2\% agarose gel electrophoresis of PCR products based on $100 \mathrm{ng}$ of total RNA isolated from rat forebrain. cDNA was used to check for the amplification of a $409 \mathrm{bp}$ fragment of the mammalian target of rapamycin (mTOR) transcript. Cyclophilin A (351 bp fragment) was used as a reference gene. Lane 1: sample from xenon-treated animal and lane 2: sample from control animal.

kinase/extracellular signal-regulated kinase (MEK)-extracellular signal-regulated protein kinase (ERK)-MAP kinaseinteracting serine threonine kinase $(\mathrm{MNK})$ signalling pathway $[8,15]$.

Support for HIF- $1 \alpha$ activation independent of mTOR or other messengers is increased because HIF- $1 \alpha$ has an internal ribosomal entry site that allows for cap-independent translation [30]. At the cellular membrane, xenon has been shown to require the opening of plasmalemmal adenosine triphosphate-sensitive potassium channels to induce neuronal preconditioning that have been shown to activate ERK [31], one of the downstream effectors of the MAPK signalling pathway. The protective effects of increased HIF$1 \alpha$ translocation demonstrated in neurons of the striatum are similar to the protective effects of xenon in developmental neuroapoptosis in rat and mouse brain [16, 17]. In mammalian cells, the expression of the $\alpha$ subunit of HIF- $1 \alpha$ is maintained at low levels under normoxic conditions but is elevated during hypoxia, when HIF- $1 \alpha$ undergoes protein stabilization and translocates from the cytoplasm into the nucleus, dimerizes with HIF- $1 \beta$, and induces the transcription of a broad range of genes involved in increasing oxygen availability and facilitating acclimatization to low oxygen level conditions [32]. Given that xenon exposure resulted in upregulation of HIF- $1 \alpha$ under normoxic conditions, it is possible that xenon enables cells to withstand hypoxic conditions through signalling cascades that are not triggered by decreases in cellular oxygen tension. For example, HIF- $1 \alpha$ has been shown to be regulated by other stimuli, including mechanical stress and growth factors [33]. The effects of the 2.3 -fold upregulation in HIF- $1 \alpha$ upon exposure to xenon observed under normoxic conditions in this study need to be further studied and may include neuroprotection, as has been shown in the setting of hypoxia-ischemia [6] that results in endogenous HIF- $1 \alpha$ upregulation.

Although this preliminary study provides novel results that differ from previously published works, it has a few important limitations. Firstly, the evaluation of other HIF$1 \alpha$ responsive genes in addition to mTOR (e.g., vascular endothelial growth factor) would have further supported a role for xenon exposure in upregulating HIF-1 $\alpha$. Furthermore, Western blot analysis of HIF- $1 \alpha$ would have provided more sensitive information on posttranslational protein levels. Although the molecular effects of xenon on HIF- $1 \alpha$ are modulated by the level of oxygenation, the investigation of different oxygen levels would have complicated the original scope of the present study.

\section{Conclusions}

In conclusion, xenon exposure during conditions of delayed preconditioning induced expression of HIF- $1 \alpha$ mRNA, which was confirmed at the intracellular level, via molecular mechanisms possibly independent of mTOR signalling. Results of future studies after the current preclinical investigation may confirm the potential benefits of xenon as an anesthetic in the context of preconditioning and neuroprotection in brain ischemia.

\section{Abbreviations}

ADNP: Activity-dependent neuroprotective protein

APC: $\quad$ Anesthetic-induced preconditioning

cDNA: Complementary DNA

DIG: Digoxigenin

DNA: Deoxyribonucleic acid

ERK: $\quad$ Extracellular signal-regulated protein kinase

G3PDH: Glyceraldehyde-3-phosphate dehydrogenase

HIF-1 $\alpha$ : Hypoxia inducible factor 1 alpha

MAPK: Mitogen-activated protein kinase

MEK: $\quad$ Mitogen-activated protein

kinase/extracellular signal-regulated kinase

MNK: $\quad$ MAP kinase-interacting serine threonine

kinase

mTOR: Mammalian target of rapamycin

P7: $\quad$ Postnatal day 7

PI3K: $\quad$ Phosphoinositide 3-kinase

PI5P4K- $\beta$ : Phosphatidylinositol-5-phosphate 4-kinase $\beta$ subtype

RNA: Ribonucleic acid 


\section{RT-PCR: Reverse transcription polymerase chain reaction \\ SSH: Suppression subtractive hybridization.}

\section{Acknowledgment}

This work was supported by the Department of Surgery, University of Pisa School of Medicine, Pisa, Italy.

\section{References}

[1] H. M. Homi, N. Yokoo, D. Ma et al., "The neuroprotective effect of xenon administration during transient middle cerebral artery occlusion in mice," Anesthesiology, vol. 99, no. 4, pp. 876-881, 2003.

[2] D. Ma, S. Wilhelm, M. Maze, and N. P. Franks, "Neuroprotective and neurotoxic properties of the "inert" gas, xenon," British Journal of Anaesthesia, vol. 89, no. 5, pp. 739-746, 2002.

[3] D. Ma, H. Yang, J. Lynch, N. P. Franks, M. Maze, and H. P. Grocott, "Xenon attenuates cardiopulmonary bypass-induced neurologic and neurocognitive dysfunction in the rat," Anesthesiology, vol. 98, no. 3, pp. 690-698, 2003.

[4] C. Petzelt, P. Blom, W. Schmehl, J. Müller, and W. J. Kox, "Prevention of neurotoxicity in hypoxic cortical neurons by the noble gas xenon," Life Sciences, vol. 72, no. 17, pp. 1909-1918, 2003.

[5] S. Wilhelm, D. Ma, M. Maze, and N. P. Franks, "Effects of xenon on in vitro and in vivo models of neuronal injury," Anesthesiology, vol. 96, no. 6, pp. 1485-1491, 2002.

[6] D. Ma, M. Hossain, A. Chow et al., "Xenon and hypothermia combine to provide neuroprotection from neonatal asphyxia," Annals of Neurology, vol. 58, no. 2, pp. 182-193, 2005.

[7] D. Ma, M. Hossain, G. K. J. Pettet et al., "Xenon preconditioning reduces brain damage from neonatal asphyxia in rats," Journal of Cerebral Blood Flow \& Metabolism, vol. 26, no. 2, pp. 199-208, 2006.

[8] N. C. Weber, O. Toma, J. I. Wolter, N. M. Wirthle, W. Schlack, and B. Preckel, "Mechanisms of xenon- and isoflurane-induced preconditioning - a potential link to the cytoskeleton via the MAPKAPK-2/HSP27 pathway," British Journal of Pharmacology, vol. 146, no. 3, p. 445, 2005.

[9] K. Julier, R. da Silva, C. Garcia et al., "Preconditioning by sevoflurane decreases biochemical markers for myocardial and renal dysfunction in coronary artery bypass graft surgery: a double-blinded, placebo-controlled, multicenter study," Anesthesiology, vol. 98, no. 6, pp. 1315-1327, 2003.

[10] J. R. Kersten, T. J. Schmeling, P. S. Pagel, G. J. Gross, and D. C. Warltier, "Isoflurane mimics ischemic preconditioning via activation of K(ATP) channels: reduction of myocardial infarct size with an acute memory phase," Anesthesiology, vol. 87, no. 2, pp. 361-370, 1997.

[11] H. J. Steiger and D. Hänggi, "Ischaemic preconditioning of the brain, mechanisms and applications," Acta Neurochirurgica, vol. 149, no. 1, pp. 1-10, 2007.

[12] M. Uecker, R. Da Silva, T. Grampp, T. Pasch, M. C. Schaub, and M. Zaugg, "Translocation of protein kinase $\mathrm{C}$ isoforms to subcellular targets in ischemic and anesthetic preconditioning," Anesthesiology, vol. 99, no. 1, pp. 138-147, 2003.

[13] F. R. Sharp, R. Ran, A. Lu et al., "Hypoxic preconditioning protects against ischemic brain injury," NeuroRx, vol. 1, no. 1, pp. 26-35, 2004.
[14] A. B. Stein, X. L. Tang, Y. Guo, Y. T. Xuan, B. Dawn, and R. Bolli, "Delayed adaptation of the heart to stress: late preconditioning," Stroke, vol. 35, no. 11, pp. 2676-2679, 2004.

[15] X. Q. Liu, R. Sheng, and Z. H. Qin, "The neuroprotective mechanism of brain ischemic preconditioning," Acta Pharmacologica Sinica, vol. 30, no. 8, pp. 1071-1080, 2009.

[16] D. Cattano, P. Williamson, K. Fukui et al., "Potential of xenon to induce or to protect against neuroapoptosis in the developing mouse brain," Canadian Journal of Anesthesia, vol. 55, no. 7, pp. 429-436, 2008.

[17] D. Ma, P. Williamson, A. Januszewski et al., "Xenon mitigates isoflurane-induced neuronal apoptosis in the developing rodent brain," Anesthesiology, vol. 106, no. 4, pp. 746-753, 2007.

[18] J. Dingley, J. Tooley, H. Porter, and M. Thoresen, "Xenon provides short-term neuroprotection in neonatal rats when administered after hypoxia-ischemia," Stroke, vol. 37, no. 2, pp. 501-506, 2006.

[19] D. Cattano, S. Valleggi, D. Ma et al., "Xenon induces transcription of ADNP in neonatal rat brain," Neuroscience Letters, vol. 440, no. 3, pp. 217-221, 2008.

[20] S. Valleggi, A. O. Cavazzana, R. Bernardi et al., "Xenon upregulates several genes that are not up-regulated by nitrous oxide," Journal of Neurosurgical Anesthesiology, vol. 20, no. 4, pp. 226-232, 2008.

[21] G. L. Semenza, "Regulation of mammalian $\mathrm{O}_{2}$ homeostasis by hypoxia-inducible factor 1," Annual Review of Cell and Developmental Biology, vol. 15, pp. 551-578, 1999.

[22] L. Swiech, M. Perycz, A. Malik, and J. Jaworski, "Role of mTOR in physiology and pathology of the nervous system," Biochimica et Biophysica Acta, vol. 1784, no. 1, pp. 116-132, 2008.

[23] D. Ma, T. Lim, J. Xu et al., "Xenon preconditioning protects against renal ischemic-reperfusion injury via hif- $1 \alpha$ activation," Journal of the American Society of Nephrology, vol. 20, no. 4, pp. 713-720, 2009.

[24] M. Bernaudin, Y. Tang, M. Reilly, E. Petit, and F. R. Sharp, "Brain genomic response following hypoxia and re-oxygenation in the neonatal rat: identification of genes that might contribute to hypoxia-induced ischemic tolerance," The Journal of Biological Chemistry, vol. 277, no. 42, pp. 39728-39738, 2002.

[25] D. Cattano, S. Valleggi, A. O. Cavazzana et al., "Xenon exposure in the neonatal rat brain: effects on genes that regulate apoptosis," Minerva Anestesiologica, vol. 77, no. 6, pp. 571-578, 2011.

[26] N. M. Jones, E. M. Lee, T. G. Brown, B. Jarrott, and P. M. Beart, "Hypoxic preconditioning produces differential expression of hypoxia-inducible factor- $1 \alpha$ (HIF- $1 \alpha)$ and its regulatory enzyme HIF prolyl hydroxylase 2 in neonatal rat brain," Neuroscience Letters, vol. 404, no. 1-2, pp. 72-77, 2006.

[27] C. Grimm, A. Wenzel, N. Acar, S. Keller, M. Seeliger, and M. Gassmann, "Hypoxic preconditioning and erythropoietin protect retinal neurons from degeneration," Advances in Experimental Medicine and Biology, vol. 588, pp. 119-131, 2006.

[28] V. Limatola, P. Ward, D. Cattano et al., "Xenon preconditioning confers neuroprotection regardless of gender in a mouse model of transient middle cerebral artery occlusion," Neuroscience, vol. 165, no. 3, pp. 874-881, 2010.

[29] N. Pore, Z. Jiang, H. K. Shu, E. Bernhard, G. D. Kao, and A. Maity, "Aktl activation can augment hypoxia-inducible factor- $1 \alpha$ expression by increasing protein translation through a mammalian target of rapamycin-independent pathway," Molecular Cancer Research, vol. 4, no. 7, pp. 471-479, 2006.

[30] K. J. D. Lang, A. Kappel, and G. J. Goodall, "Hypoxia-inducible factor- $1 \alpha$ mRNA contains an internal ribosome entry site that 
allows efficient translation during normoxia and hypoxia," Molecular Biology of the Cell, vol. 13, no. 5, pp. 1792-1801, 2002.

[31] L. Huang, B. Li, W. Li, H. Guo, and F. Zou, "ATP-sensitive potassium channels control glioma cells proliferation by regulating ERK activity," Carcinogenesis, vol. 30, no. 5, pp. 737-744, 2009.

[32] A. O. Fedele, M. L. Whitelaw, and D. J. Peet, "Regulation of gene expression by the hypoxia-inducible factors," Molecular Interventions, vol. 2, no. 4, pp. 229-243, 2002.

[33] Y. S. Chun, M. S. Kim, and J. W. Park, "Oxygen-dependent and -independent regulation of HIF-1alpha," Journal of Korean Medical Science, vol. 17, no. 5, pp. 581-588, 2002. 


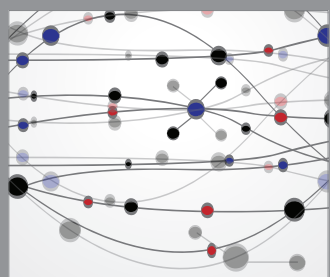

The Scientific World Journal
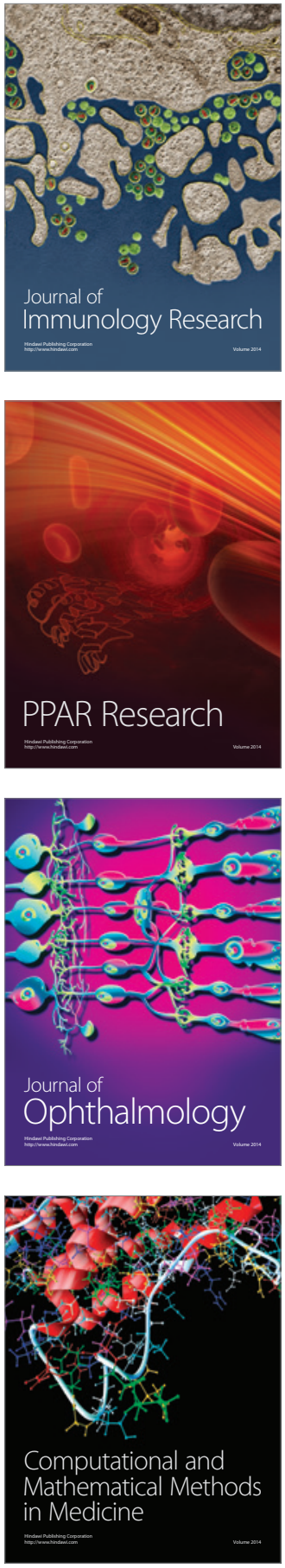

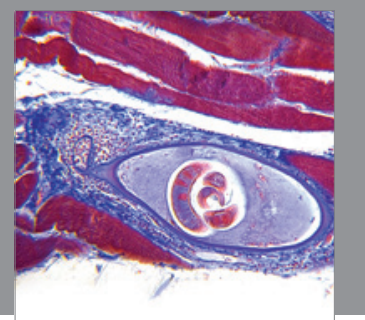

Gastroenterology

Research and Practice
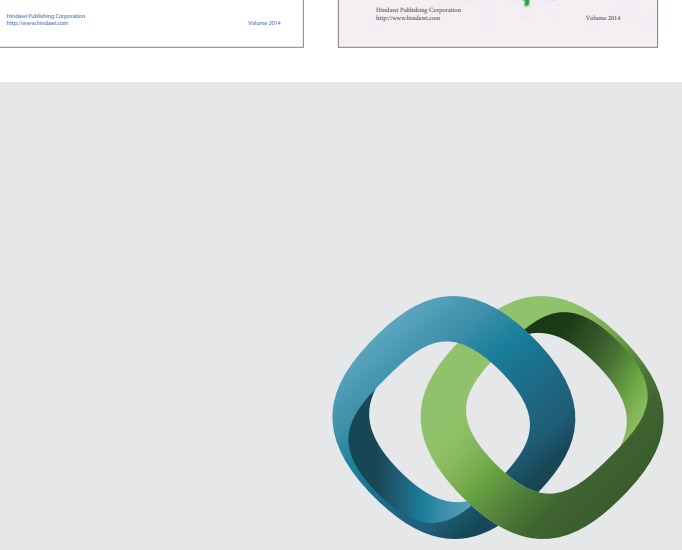

\section{Hindawi}

Submit your manuscripts at

http://www.hindawi.com
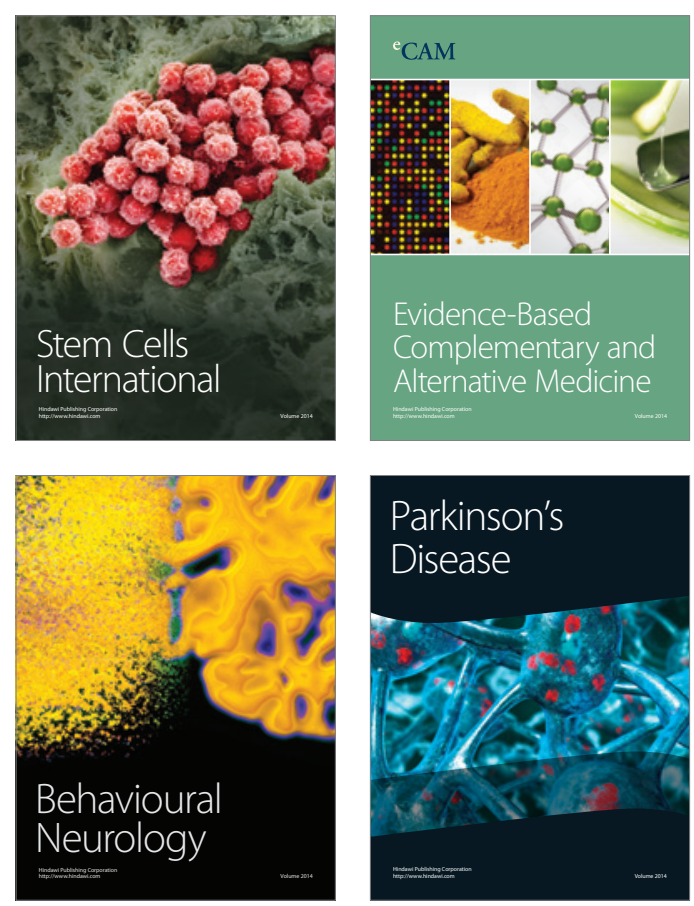

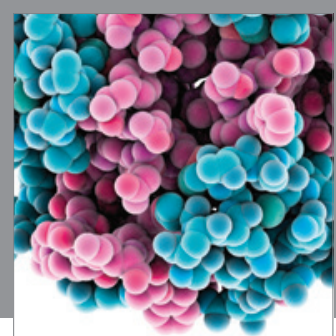

Journal of
Diabetes Research

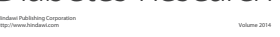

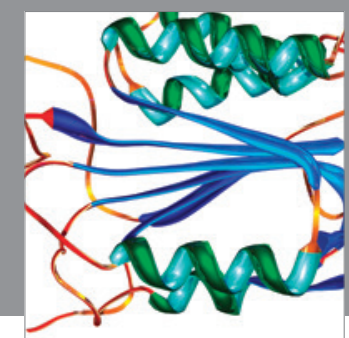

Disease Markers
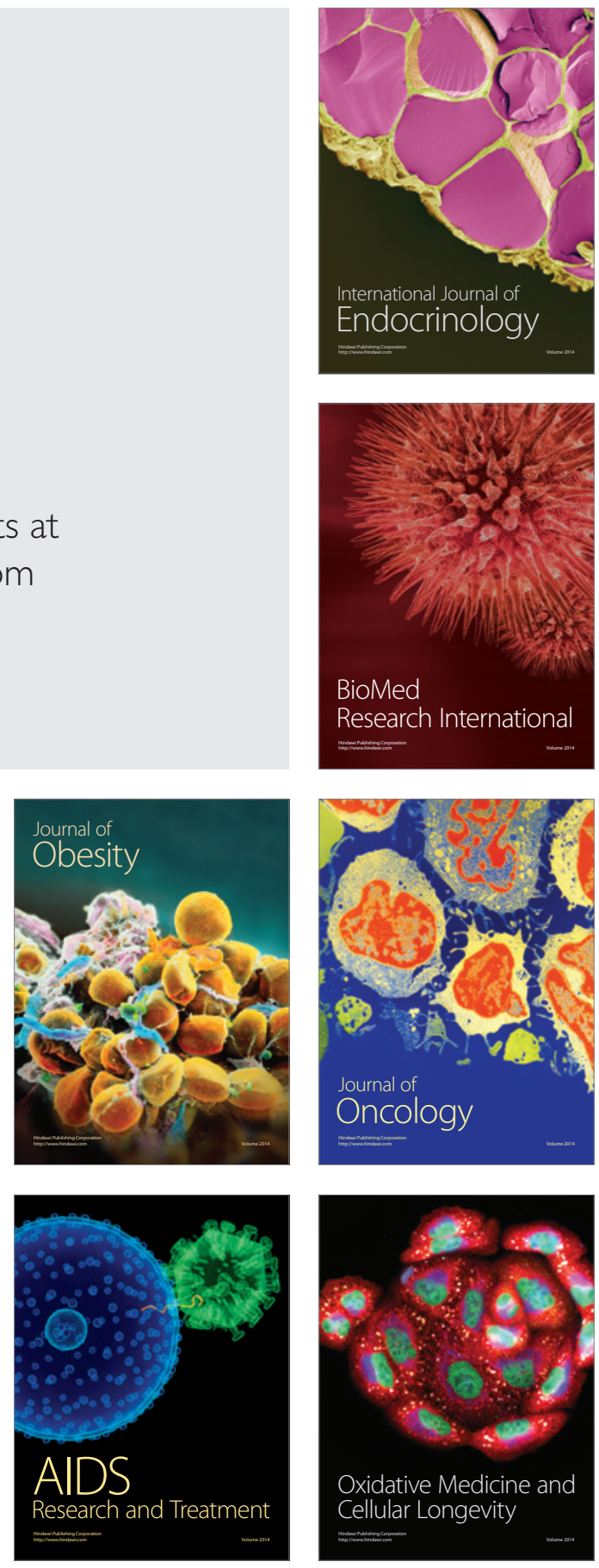